\section{Découverte de la nesfatine-1}

Jacques Epelbaum
Inserm UMR 549, Faculté de Médecine,

Université Paris Descartes,

IFR Broca-Sainte Anne,

2 ter, rue d'Alésia, 75014 Paris, France.

epelbaum@broca.inserm.fr

neurones. Ils montrèrent ensuite que, comme l'expression de NUCB2, les concentrations de nesfatine-1 dans le noyau paraventriculaire de l'hypothalamus étaient considérablement et sélectivement diminuées après un jeûne de 24 heures. Une injection intracérébroventriculaire journalière de nesfatine-l pendant dix jours entraîna une baisse considérable de la prise alimentaire (plus de $30 \%$ ) et un retard dans la croissance pondérale. Des effets en miroir furent observés lorsque des morpholinos antisens de NUCB2 furent injectés dans le même protocole. Enfin, les auteurs recherchèrent les interactions éventuelles avec les systèmes déjà impliqués dans le contrôle de la prise alimentaire. Chez le rat Zucker qui présente un récepteur leptine inactif, la nesfatine-l s'avéra toujours active. En revanche, l'injection intracérébroventriculaire d'un antagoniste des récepteurs des mélanocortines de type MC3/4, le SHU9119, abolit l'effet satiétogène de la protéine

Les auteurs concluent un peu rapidement qu'ils ont découvert une nouvelle cible pour le traitement de l'obésité. II reste en effet à déterminer le mécanisme d'action de cette nouvelle protéine puisque, de façon un peu surprenante, l'injection de nesfatine-l ne semble modifier l'expression d'aucun des peptides hypothalamiques connus pour intervenir dans le contrôle de la prise alimentaire et testés par les auteurs, tels le neuropeptide Y, l’AgRP, le CRH, les dérivés de la POMC. II est vrai qu'il reste d'autres candidats... $\diamond$

Identification of nesfatin- 1

\section{RÉFÉRENCES}

1. Oh-I S, Shimizu H, Satoh T, et al. Identification of nesfatin-1 as a satiety molecule in the hypothalamus Nature 2006 ; $443: 709-12$.

2. Steppan CM, Balley ST, Bhat $S$, et al. The hormone resistin links obesity to diabetes. Nature 2001 ; 409 : 307-12.

3. Wiesner G, Morash BA, Ur $\varepsilon$, et al. Food restriction regulates adipose-specific cytokines in pituitary gland but not in hypothalamus. J Endocrinol $2004 ; 180$ : Rl-6. 